\title{
The Application of Short-Term Deterministic Wave Prediction to Offshore Electricity Generation
}

\author{
J.R.Halliday ${ }^{1}$, D.G. Dorrell ${ }^{1}$ and A.R. Wood ${ }^{2}$ \\ ${ }^{1}$ Department of Electronics and Electrical Engineering \\ University of Glasgow, Oakfield Avenue, Glasgow, G12 8LT, UK \\ Tel: +44 141330 2768, email: r.halliday@elec.gla.ac.uk, d.dorrell@elec.gla.ac.uk \\ ${ }^{2}$ Dept of Electrical and Computer Engineering \\ University of Canterbury, Private Bag 4800, Christchurch, New Zealand \\ Tel: +64 (3) 3642-406 ext:6406, email: a.wood@elec.canterbury.ac.nz
}

\begin{abstract}
The field of wave energy extraction from the oceans is moving from the realm of drawing board dreams to the reality of device farms in the open ocean. Several devices are now at the stage of full scale testing and methods of increasing their economic potential are required. Maximum energy capture from a given sea state is one such way of increasing production and for devices to achieve this an accurate prediction of the sea surface elevation in the vicinity of these devices is required. The method proposed is based on the work of Belmont [1] and Zhang [2], extending from unidirectional modelling to directional. Preliminary simulations for a unidirectional buoy excited by a Pierson-Moskowitz spectrum are given and the proposed method for directional modelling is then set forth.
\end{abstract}

\section{Key words}

Wave Energy, Wave Prediction, Wave Modelling.

\section{Introduction}

For the past 30 years the field of wave energy has been gently progressing in the somewhat disjointed grouping of renewable energy research. The field was put into the spotlight during the oil crisis of the mid 1970's when Europe grew edgy over its dependence on Middle Eastern oil and looked for other means of generating electricity.

Before wind energy was given serious consideration the oceans, in particular the North Atlantic and to a lesser extent the Mediterranean Sea, were seen as valuable resource. The energy off the West Coast of Scotland has some $50 \mathrm{~kW} / \mathrm{m}$ of wave front [3], with similar levels off the coasts of Ireland, France, Spain and Portugal. Many ideas proliferated during this time, but few went on to be developed and even fewer reached full scale implementation.

One of the greatest problems faced by the device teams was in making the devices strong enough to survive harsh offshore conditions while still being able to extract enough energy to make them economical. Of the devices developed in this era were Salter's Ducks, The Bristol Cylinder, The Cockerell Raft and many others which are described in [4].

The enthusiasm of the 1970's quickly waned as the price of oil fell and North Sea oil and gas came online. With a cheap and apparently plentiful supply of energy the plans of various governments for wave devices were dropped [5]. Interest has been renewed in the last decade with the rise of public interest in environmental concerns. Governments in Europe, recognising the political capital that could be gained from giving incentives to renewables, began funding various programs.

Incentives, such as the Kyoto agreement, have led to the setting of sweeping targets of renewable generation that some governments are committed to meet. For the UK the target varies between 15 and $40 \%$, depending on which group the politician delivering their speech is addressing. The setting of the targets to be met has led an abundance of wind energy plants being erected. Wind energy is seen by the public as being green and the costs to the electricity companies are lower than for other devices. Whilst providing a suitably green image, the encroachment of windmills is beginning to be questioned by many concerned with the effects they will have on the countryside and particularly the tourism economy on which many rural areas depend. The dependence of large parts of the national grid on wind energy can also lead to shortfalls in supply with energy needing to be drawn from other countries.

Some of the money and incentives for renewable generation has trickled through to the field of wave energy and has been put to good use by the device teams based in Europe. Wavegen has developed the LIMPIT device and has plans for a redeployment of the OSPREY in the near future. The WaveDragon is undergoing sea trials, as is the Archimedes Wave Swing. The device on which the remainder of this paper will focus is the Pelamis (described in Section 2C).

Section 2 will give an overview of the short term wave prediction problem. Section 3 will give a basic description of the field of wave modelling. The proposed prediction method will be stated in Section 4 with a worked example and results in Section 5. The paper will finish with the conclusions of this initial research.

\section{Short term wave prediction}

\section{A. The European Wave Energy Network (EWEN)}

In 2003 a report was published by the European Wave Energy Network [6] described the work conducted on wave energy in the European Union. It covered areas such as farm siting, economic support for devices and future developments that would be required for wave energy to become profitable.

One of the areas which were recognised as requiring work was in the short term prediction of wave behaviour. Medium to Long term prediction is from $1 \mathrm{hr}$ to 1 week 
ahead and is already a well developed field. The short term is from $10 \mathrm{~s}$ to $1 \mathrm{~min}$ ahead giving an accurate prediction of the surface elevation at a point on the sea surface.

Many of the devices which are being developed contain features which allow them to selectively change the frequency range over which they operate. A device such as an oscillating water column, on which much has been published, is essentially a large concrete box that operates at a fixed resonant frequency. Devices that float on the surface of the sea will also have a resonant frequency related to the specific dimensions of the device. For an oscillating water column this frequency can be changed by varying the pressure in the caisson by way of valves. For floating structures this is accomplished by varying fluid pressures in the mechanical connections of the device. In order to properly tune this resonance accurate information on the next expected wave is required. It is this information that is at present unavailable.

\section{B. Prior Work}

Work on short term prediction has taken place in the past with significant contributions from Zhang [2] and Belmont [1]. Zhang's 1997 paper demonstrated that a complex wave field can be decomposed into a specific set of wave trains using a maximum least likelihood approach. The resultant set of wave trains could then be used in making predictions of wave height in the vicinity of the measurements. The study showed that it was vitally important to retrieve the phase information for each wave train as well as the amplitude and frequency in order to make an accurate prediction of the surface elevation.

Belmont [1] went further with this method and has applied it to the prediction of wave elevation for use in the controlled landing of helicopters on ships. The main result of the paper is that a prediction can be made up to 30 seconds ahead from the point of data sampling. The method involved was based on the Discrete Fourier Transform and an adaptive windowing method of end fitting the raw sampled data.

\section{The Pelamis}

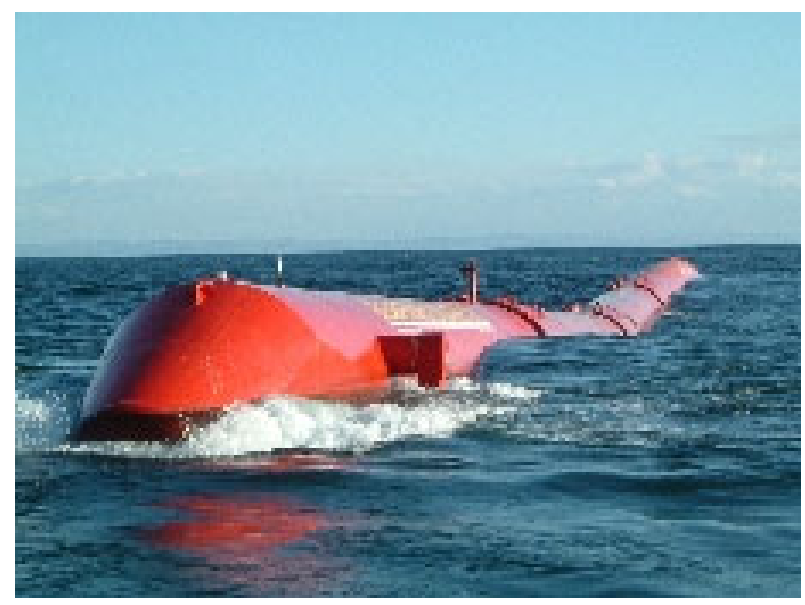

Fig. 1. A view of the Pelamis device
The Pelamis device has been developed by Ocean Power Delivery Ltd., a company based in Edinburgh and arose from the $\mathrm{PhD}$ theses of several students in the Wave Energy group at the University of Edinburgh. The device is shown in Fig. 1 and consists of four cylindrical sections joined at theirs ends by hydraulic rams. Its dimensions are $150 \mathrm{~m}$ in length and $3.5 \mathrm{~m}$ in diameter. The device was designed with survivability as its main goal and achieves this by being oriented lengthways into the prevailing wave direction and has the ability to de-reference itself in extreme wave conditions by its ability to duck underneath the highest of waves in a manner similar to a surfer getting out beyond the breakers on a beach. The resonance of the device can be varied by changing the resistance of the rams joining the sections. The resistance can be changed on a wave to wave basis and so that short term forecast will be of benefit.

Testing for the device has taken place of several small-scale models and at present a full-size test is ongoing at the EMEC test centre on the Orkney Islands. A possible farm design is planned for the Azores and it is at this stage of development that this research will focus on. Each device requires $150 \mathrm{~m}$ by $200 \mathrm{~m}$ of space in which to operate, so a 4-6 device farm would require $600-900 \mathrm{~m}$ by $200 \mathrm{~m}$. The device has an optimum operating range for waves with a time period of 5 to 13 seconds, with a maximum output of $750 \mathrm{~kW}$ in seas of $5 \mathrm{~m}$ amplitude. It is envisaged that the device will be moored at the $50 \mathrm{~m}$ contour.

TABLE 1. Valid prediction distances at 30s

\begin{tabular}{|c|c|c|c|}
\hline Wavelength & Time Period & $\begin{array}{c}\text { Wave } \\
\text { Velocity }\end{array}$ & $\begin{array}{c}\text { Valid } \\
\text { Distance }\end{array}$ \\
\hline $39 \mathrm{~m}$ & $4.99 \mathrm{~s}$ & $7.81 \mathrm{~m} / \mathrm{s}$ & $234 \mathrm{~m}$ \\
\hline $100 \mathrm{~m}$ & $8.00 \mathrm{~s}$ & $12.5 \mathrm{~m} / \mathrm{s}$ & $375 \mathrm{~m}$ \\
\hline $150 \mathrm{~m}$ & $9.8 \mathrm{~s}$ & $15.3 \mathrm{~m} / \mathrm{s}$ & $459 \mathrm{~m}$ \\
\hline $200 \mathrm{~m}$ & $11.3 \mathrm{~s}$ & $17.69 \mathrm{~m} / \mathrm{s}$ & $530 \mathrm{~m}$ \\
\hline $250 \mathrm{~m}$ & $12.65 \mathrm{~s}$ & $19.76 \mathrm{~m} / \mathrm{s}$ & $592 \mathrm{~m}$ \\
\hline
\end{tabular}

From Belmont [1] a valid prediction window of $30 \mathrm{sec}$ ahead can be assumed for waves in the range in which the Pelamis is designed to operate. Using deep water wave equations and a range of $39-250 \mathrm{~m}$, a valid prediction distance of $230 \mathrm{~m}$ from the point of sampling can be assumed for the shortest wavelength of interest to the device, given in Table 1. This should be a great enough distance to cover a device farm of 4-6 devices.

\section{Measurement}

Though the two methods used by Zhang and Belmont show that short term prediction is possible, the way in which the data was recorded in each of the papers is not economically viable for a wave farm.

In Zhang's experiments the data was recovered from an array of pressure sensors sited on an offshore platform. For a wave farm the cost of building an additional structure for the mounting of sensors is prohibitive. Pressure sensors are a possible alternative, but at a depth of $50 \mathrm{~m}$ will lose their accuracy at higher frequencies. The use of an array of wave rider buoys, recording the surface elevation is also an option but again the cost of sighting many buoys is expensive and 
they would also pose a possible hazard if they were to break free.

Belmont's research uses a forward scanning LIDAR device. The LIDAR would be mounted onboard the ship on which a helicopter is to land and be orientated to face the direction of prevailing swell. At present the LIDAR device is able to distinguish a unidirectional wave train from a mixed sea and make a prediction. Unfortunately for an offshore wave farm there is no structure on which to mount a LIDAR and as with Zhang's method would prove uneconomical. This being said a LIDAR mounted on a shore-based device would be most useful.

In order to prove useful to the wave energy community this research focuses on existing measurement techniques using tried and tested equipment that is widely available. The goal is to make a prediction based on the readings of a directional wave buoy, which can be relatively cheaply moored and serviced.

\section{Wave modelling}

\section{A. The basics}

Interest in the modelling and prediction of the waves has existed since early civilisation but scientific study did not truly begin until after the Second World War when the landing of troops on the Normandy beaches was affected by the sea conditions. In the UK research took place at the National Institute of Oceanography, which was to become part of Southampton Institute of Oceanography.

A key realisation made by the scientists was that a complex sea can be approximated as the linear superposition of a large number of long crested periodic wave trains of low amplitude with different frequencies and directions of propagation all travelling independently of each other, A mathematical representation is given in Eqn. 1.

$$
\zeta(x, y, t)=\sum_{n=1}^{\infty} a_{n} \sin \left(k_{n} x \cos \theta_{n}+k_{n} y \sin \theta_{n}-\omega_{n} t-\phi_{n}\right)
$$

where the subscript $n$ refers to the $n^{\text {th }}$ component, $\zeta$ is the surface elevation about mean sea level, $\omega_{n}$ is the angular frequency, $k_{n}$ is the wave number, $\theta_{n}$ is the direction in which the wave is travelling to and $\phi_{n}$ is the phase angle. This equation forms the basis for the analysis of wave records which are recorded by a variety of devices, such as wave buoys. The outputs of these devices can generally be transformed into a record of wave height and plotted against time. The records are then analysed, typically by means of a Discrete Fourier Transform, to give a spectral representation of a fixed time period, usually 1200 seconds. From these records various time period parameters are extracted to be used in further analysis.

\section{B. Deep water equations}

For small disturbances of the sea surface the hydrodynamic equations are linear and can be solved to give freely propagating, long-crested, sinusoidal wave trains.
In deep water (depth $h>\lambda / 4$ ) the water particles travel in circular orbits with the particles moving forwards in the crests and backwards in the troughs. If the $x$ and $z$ displacements of a particle are $\chi$ and $\zeta$ from its rest position $x$ at a depth $z$ below the surface

$$
\begin{aligned}
& \chi=a e^{k z} \cos (k x-\omega t-\phi) \\
& \zeta=a e^{k z} \sin (k x-\omega t-\phi)
\end{aligned}
$$

Where, $\omega^{2}=g k$ is the dispersion relationship, $g$ is the acceleration due to gravity, $k$ is the wave number $k=2 \pi / \lambda$, $\lambda$ is the wavelength and $\phi$ is a random phase angle $=\pi<\phi$ $<\pi$.

Equation 3 can be restated to include the effect of the direction of travel $\theta$

$$
\zeta(x, y, z)=a e^{k z} \sin (l x+m y-\omega t-\phi)
$$

where $l=k \cos \phi$ and $m=k \sin \phi$. An example showing a single wave train with $\lambda=100 \mathrm{~m}$ and $\theta=0^{\circ}$ is shown in the Fig. 2.

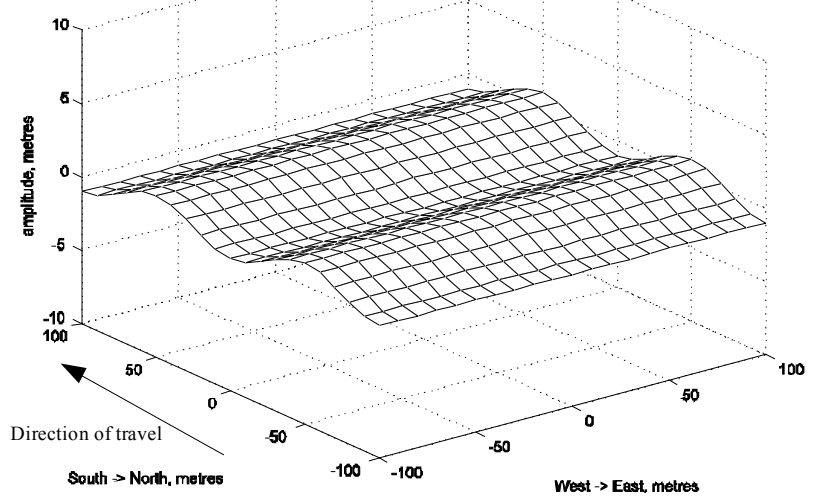

Fig. 2. A example of a wave train

\section{Airy Equations}

In the case of the Pelamis device, which is to be situated in $50 \mathrm{~m}$ depth the deep water equations will no longer strictly apply to the longer wavelengths and the Airy Equations must be used in predictions.

These equations were derived in 1845 by Sir G.B. Airy [7]. The equations for a small-amplitude sinusoidal wave in water of depth $h$ ( $h$ is taken as positive, $z$ as negative: that is the point when $z+h=0$ is the sea bed) are

$$
\begin{aligned}
& \chi=a \frac{\cosh k(z+h)}{\sinh k h} \cos (k x-\omega t-\phi) \\
& \zeta=a \frac{\sinh k(z+h)}{\sinh k h} \sin (k x-\omega t-\phi)
\end{aligned}
$$

These basic equations are all in terms of the local wave number $k$, in practise the data available is usually the period 
$T$ or the frequency $f$ as the phase velocity $c p=\lambda / T=\omega / k$, then

$$
\omega^{2}=g k \tanh k h
$$

Figs. 3 and 4 show the difference in $k$ number between the deep water equations and the Airy formulation.

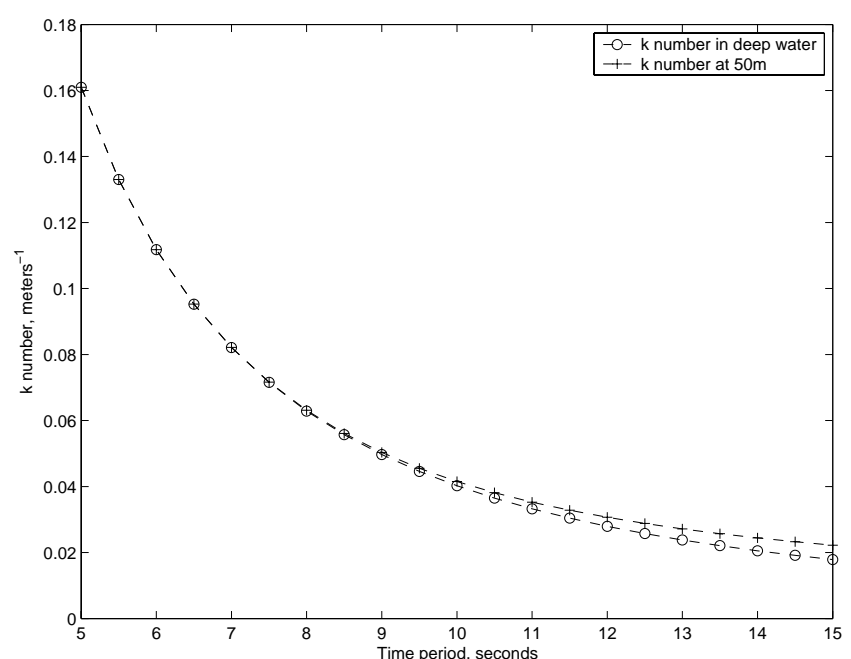

Fig. 3. $k$ number plotted against time period for deep water and airy equations at $50 \mathrm{~m}$

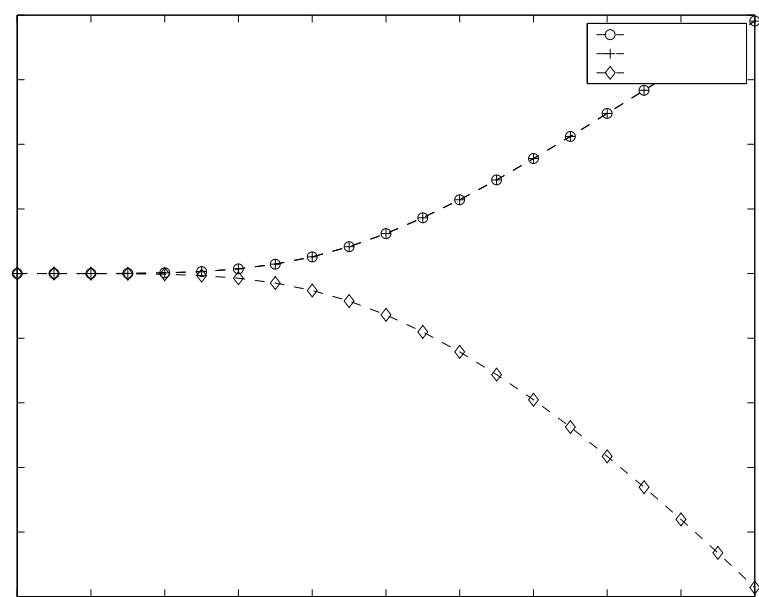

Fig. 4. Percentage deviation from deep water values of wavelength, phase velocity and $k$ plotted against time period

It can be seen that in deep water, where $\tanh (k h) \rightarrow 1$ the approximation $\omega^{2}=g k$ is valid. As the water depth decreases the longer wavelengths will begin to feel the bottom. The effect of this is to slow down the phase velocity of the wave and to some extent cause the wave amplitude to increase. The effect of this is easily seen on any shallow sloping beach where the waves can be seen increasing in height as they approach the shore before eventually breaking.

For the Pelamis device we need to use the full Airy equations as for the longer wavelengths of interest the water in which the devices are situated is too shallow for the deepwater approximation to strictly hold true. As shown above the percentage deviations from the deep-water values increase for time periods greater than $7 \mathrm{sec}$. The most significant result from above is the change in phase velocity as the wave enters the shallower water. This is important in the prediction of waves as otherwise it would be assumed that the waves of a longer time period travel further that they actually would.

\section{$D$. The omni-directional spectral density function, $S(f)$}

$S(f)$ is called "the omnidirectional spectral density function" and has units of $\mathrm{m}^{2} / \mathrm{Hz}$. It is this function that is measured by a uni-directional wave buoy, such as Datawell's Waverider. This function forms the basis of wave measurement and prediction. The following gives the derivation of this function.

If the elevation of the sea surface is measured above a fixed point, $(0,0)$, then Eqn. 4 loses its dependence on $x, y$ and reduces to

$$
\zeta(t)=\sum_{n} a_{n} \sin \left(\omega_{n} t-\phi_{n}\right)
$$

squaring this

$$
\begin{aligned}
\zeta^{2}(t) & =\sum_{n} \sum_{m} a_{n} a_{m} \sin \left(\omega_{n} t-\phi_{n}\right) \sin \left(\omega_{m} t-\phi_{m}\right) \\
\zeta^{2}(t)= & \sum_{n} \sum_{m} \frac{1}{2} a_{n} a_{m} \times \\
& \left\{\begin{array}{l}
\cos \left[\left(\omega_{n}-\omega_{m}\right) t+\left(\phi_{n}-\phi_{m}\right)\right] \\
-\cos \left[\left(\omega_{n}+\omega_{m}\right) t+\left(\phi_{n}+\phi_{m}\right)\right]
\end{array}\right\}
\end{aligned}
$$

Taking the long term average, components with $n \neq m$ become zero. Allowing $n=m$ and taking the long term average

$E=\overline{\zeta^{2}(t)}=\sum_{n} \frac{1}{2} a_{n}^{2}$

That is, the variance of the sea-surface elevation equals the sum of the variances of its component wave trains. As the variance is proportional to the average energy per unit area of the sea surface, the total energy of the wave system is just the sum of the energies associated with individual wave trains.

If the output of a wave recording device is filtered to select only those frequencies in the range $f-\Delta f / 2$ to $f+\Delta f / 2$, giving a variance $\Delta E$, then a function $S(f)$ can be defined by

$S(f)=\Delta E / \Delta f$

$S(f)$ will remain finite as $\Delta f \rightarrow 1$ and from this it can be seen that

$$
E=\int_{0}^{\infty} S(f) d f
$$

In practise it is not possible to continuously sample the sea surface elevation and an estimate has to be made for a finite 
period of time/space, this is distinguished as $\hat{S}(f)$. An example of an omni-directional Pierson-Moskowitz spectrum [11] for a fully developed sea is given in Fig. 5.

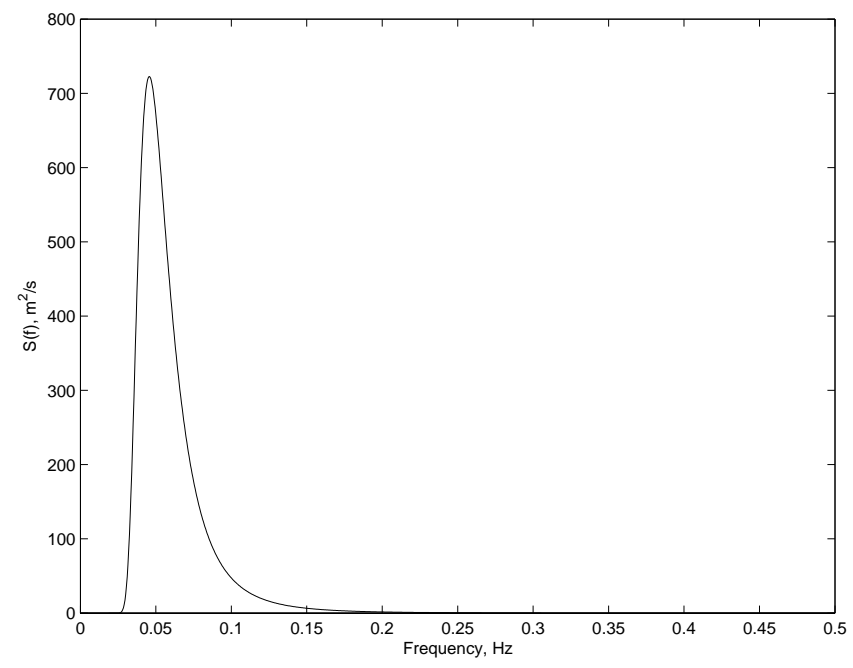

Fig. 5. Example of an omni-directional spectrum

Such spectra are derived from the Fourier transform of a recorded time series.

The estimate $\hat{S}(f)$ of the spectral density is obtained by summing the variances ("energies") of all the Fourier components within the chosen spectral resolution $\Delta f$

$\hat{S}(f) \Delta f=\frac{1}{2} \sum_{\Delta f}\left(a_{n}^{2}+b_{n}^{2}\right)$

For example, if a record $1000 \mathrm{sec}$ long were available, the harmonics would be spaced at $0.001 \mathrm{~Hz}$ intervals. If a spectral resolution of $0.01 \mathrm{~Hz}$ were required, then the harmonics would be summed in groups of 10 to give the spectral estimates.

\section{E. Spectral Moments}

Some definitions and statistical results, as used by the oceanographic community, are expressed in terms of the spectral moments of the spectral density function $S(f)$. The $n^{\text {th }}$ spectral moment is given by

$$
m_{n}=\int_{0}^{\infty} f^{n} S(f) d f
$$

The spectral moment can be thought of as a frequency scaled version of the spectral density function, with the error associated with its calculation increasing with higher orders. The $0^{\text {th }}$ spectral moment is equivalent to the variance of the spectrum and is commonly used to calculate the expected significant wave height, $H_{m 0}=4 \sqrt{m_{0}}$.

\section{F. The Directional Spectrum, $S(f, \theta)$}

Eqn. 11 can also be derived from Eqn. 1, incorporating wave direction, $\theta$. If you were now able to filter in the directional wave trains, those travelling between $\theta+\Delta \theta / 2$ and $\theta-\Delta \theta / 2$ as well as in frequency, then by analogy to Eqn. 12, a directional spectral density function $S(f, \theta)$ can be defined as

$S(f, \theta)=\frac{\Delta E}{\Delta f \Delta \theta} \rightarrow \frac{d^{2} E}{d f d \theta}$

It is usual to define $S(f, \theta)$ as the product of the omnidirectional spectrum $S(f, \theta)$ and a normalised directional distribution $G(\theta)$, that is

$S(f, \theta)=S(f) G(\theta)$

So that

$\int_{0}^{\infty} G(\theta) d \theta=1$

In fact, $G(\theta)$ varies with frequency and will therefore be represented by $G(\theta, f)$. Established methods of measurement do not give estimates of $G(\theta, f)$ directly. In order to interpret such measurements usefully, it is usually necessary to make assumptions about the shape of the directional spread. The form that is most commonly used is

$G(\theta, f)=F(s) \cos ^{2 s} \frac{1}{2}\left(\theta-\theta_{1}\right)$

where $\theta_{l}$ is the predominant direction, and $s$ is known as the shape function. $s$ is a function of frequency according to Pierson-Moskowitz [11] and $\theta_{l}$ in this paper is not frequency dependent. An example of this function is shown in Fig. 6.

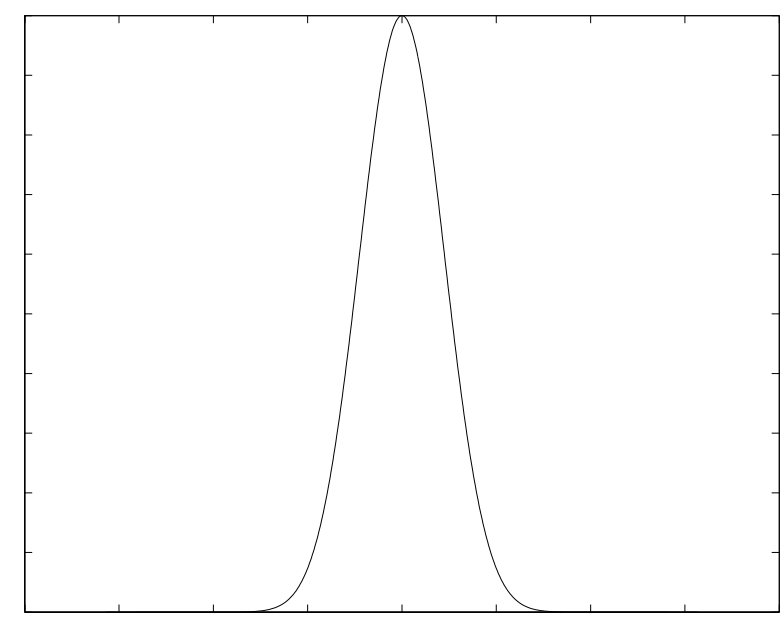

Fig. 6. Example of the directional spreading function with $s=10$

These equations were first used by D.E. Cartwright [8]. $s$ and $\theta_{l}$ are estimated from measurements. Usually two independent estimates can be obtained of each parameter at each frequency, which in principle allows a check as to whether the assumed form is correct or not. 


\section{Proposed method of prediction}

The method of prediction is to record the wave elevation time history at a fixed point, $(0,0)$. This time history is then Transformed to the frequency domain; an omni-directional spectrum created and the spectral moments stored.

The amplitudes and phases of this transform are then used to create a number of wave trains. These wave trains are then propagated to the prediction point $(0,250)$ and the time history at that point recorded. Again the time history is transformed to the frequency domain and the omnidirectional spectrum and spectral moments stored.

At the prediction point the time history and thus the spectrum and spectral moments should be available for comparison with the predicted results. Whilst having this information for two closely situated buoys would be desirable, none is available. Therefore, the data at the origin and prediction point will be derived from the same spectrum.

\section{An omni-directional prediction example}

It is proposed that a wave spectrum based on the Pierson Moskowitz [11] formulation for a fully developed sea at a wind speed of $30 \mathrm{~m} / \mathrm{s}$ will be created. This spectrum will be broken down into a number of unidirectional wave trains. The wave trains will then be used to simulate the elevation of the sea a two points, one above the origin and representing the output of the wave rider buoy and the second taken at a distance of $250 \mathrm{~m}$ from the origin and will represent the target point of prediction.

The simulated wave record taken at the origin will be examined and spectral parameters recorded. This simulated spectrum will in turn be broken down into a number of wave trains and propagated to the prediction point. A new wave record will be recorded at the prediction point. The wave record will then be compared to the expected wave record.

\section{A. The input wave trains}

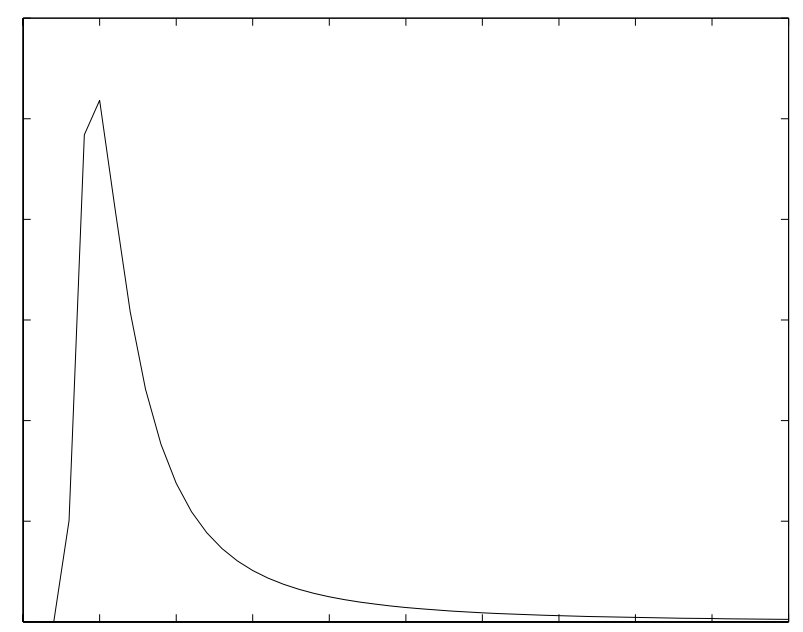

Fig. 7. Showing the amplitudes of the simulated wave trains
The spectra used to generate the input wave trains is the Pierson-Moskowitz formulation, given in Eqn. 30

$$
S(f)=\alpha_{P M} g^{2}(2 \pi)^{-4} f^{-5} \exp \left[-\beta\left(f_{0} / f\right)^{4}\right]
$$

where

$$
\begin{aligned}
& \alpha_{P M}=0.0081 \text { is empirically derived } \\
& \beta=0.74 \text { is empirically derived } \\
& f=\text { frequency } \\
& f_{0}=g /\left(2 \pi U_{19.5}\right) \text { where } U_{19.5} \text { is the wind speed } \\
& \text { recorded at } 19.5 \mathrm{~m} .
\end{aligned}
$$

The spectrum for $U_{19.5}=30 \mathrm{~m} / \mathrm{s}$ is shown in Fig. 6 for 1000 wave vectors and $\Delta f=0.0005 \mathrm{~Hz}$ and approximates to a continuous omni-directional spectrum $s(f)$.

In order to run the simulation the number of wave vectors must be reduced to 50 and $\Delta f$ increased to $0.01 \mathrm{~Hz}$ giving $\hat{S}(f)$. The equation to produce a list of amplitudes for each wave vector is:

$C_{j}=\sqrt{\hat{S}\left(f_{j}\right) \Delta f}$

This is shown graphically in Fig. 7.

From this list of amplitudes and frequencies the full Airy equations, Section 3.C, must be used in calculating the wavelengths, wave numbers and phase velocities of each component.

In addition to the spectra, the amplitudes, frequencies, spectral moments and derived time period parameters are also calculated for stored for later comparison. The discrete version of Equation 14 is

$$
\hat{m}_{p}=1 / 2 \sum_{n} f_{n}{ }^{p} c_{n}{ }^{2}
$$

\section{B. Data Generation}

Wave records taken in the field are often standardised to be 1200s in length and sampled at $3.4133 \mathrm{~Hz}$ to give 4096 samples. This is the pattern that will be used here.

The wave vector file generated in the previous section is opened and the parameters loaded in the MATLAB work space. A file containing the position of the sensors is also opened. For the sensor at $(0,0)$, the recording point, and $(250,0)$, the prediction point, the wave records are calculated using Eqn. 1.

The phase component $\phi_{n}$ is taken as being random and to some extent will simulate the random nature varying nature of the ocean, at a later time the effect of sea bed bathymetry and wave-wave interactions will also need to be modelled. The record for buoy 1 is generated from -1200 to 0 seconds, in order for it to have been a past record. The record at the prediction point is taken from 0 to 1200 seconds and will become the target wave record which we will be attempting to reproduce. 


\section{Analysis and Prediction}

The first stage in processing a wave record is to filter out any low frequency components relating to tidal patterns. Filtering is also required to remove any high-frequency components of the wave record. In Scottish waters $0.5 \mathrm{~Hz}$ is a generally accepted cut-off frequency.

The time series is analysed using an FFT into 4096 frequency components at $0.0008333 \mathrm{~Hz}$ intervals. The negative frequencies are folded into the positive half of the spectrum reducing the number of frequency bins to 2048 with an upper frequency limit of $1.7 \mathrm{~Hz}$.

The complex amplitude and phase data for the record taken at the origin is shown in Fig. 8.
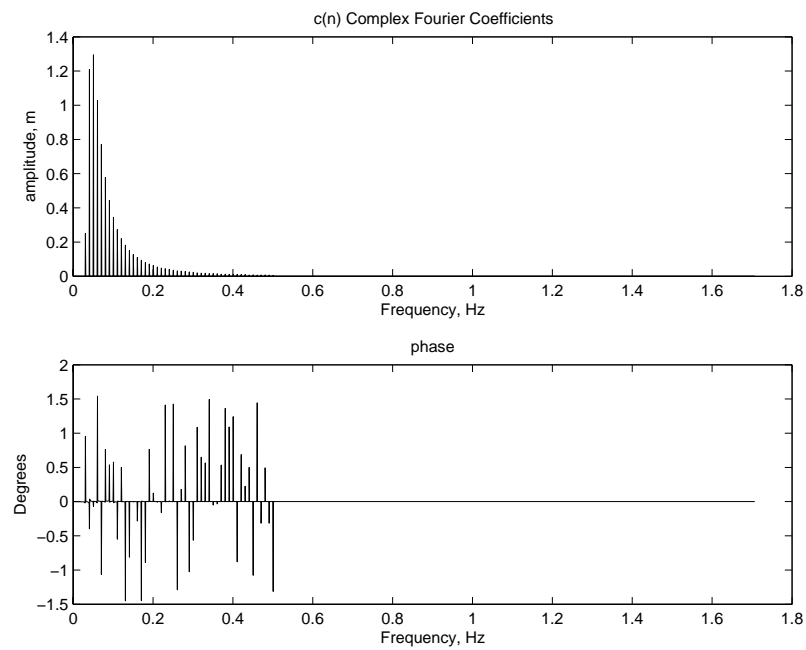

Fig. 8 The complex amplitude and phase information for the origin wave record

In the next stage of prediction the amplitude and phase information taken at the origin is used with the Airy wave equations, Section $3 C$, to create a sequence of wave trains. These wave trains are then used to calculate the time history for the prediction point. The spectra and spectral moments are then calculated with the resultant spectra shown in Fig. 9.

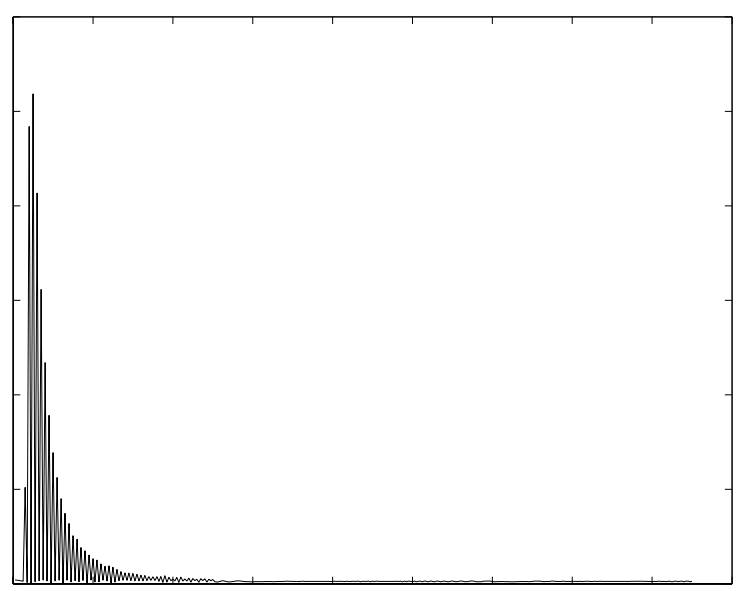

Fig. 9. Resultant omni-directional spectra for prediction point

\section{Results}

Tables 2 and 3 show the results for the comparison of various spectral parameters taken from the target spectrum and the one predicted using the previous method.

TABLE 2. Spectral moment comparisons

\begin{tabular}{|c|c|c|c|c|}
\hline & M0 & M1 & M2 & M3 \\
\hline Target & 11.51 & 0.68 & 0.047 & 0.0041 \\
\hline Prediction & 4.179 & 0.425 & 0.075 & 0.021 \\
\hline Error \% & 63.69 & 37.5 & -59.57 & -412.2 \\
\hline
\end{tabular}

TABLE 3. Time period comparisons

\begin{tabular}{|c|c|c|c|c|c|}
\hline & Hm0 & Tz & T1 & TE & Tc \\
\hline Target & 13.573 & 15.652 & 0.059 & 18.78 & 11.214 \\
\hline Prediction & 8.177 & 7.443 & 0.102 & 15.09 & 3.603 \\
\hline Error \% & 39.75 & 52.45 & -72.88 & 19.65 & 67.87 \\
\hline
\end{tabular}

As can be seen the results are not great. In [9] the errors associated with the discretizing of spectral moments were found to be great when compared to continuous spectra and this is possibly not the best of methods for comparing prediction results.

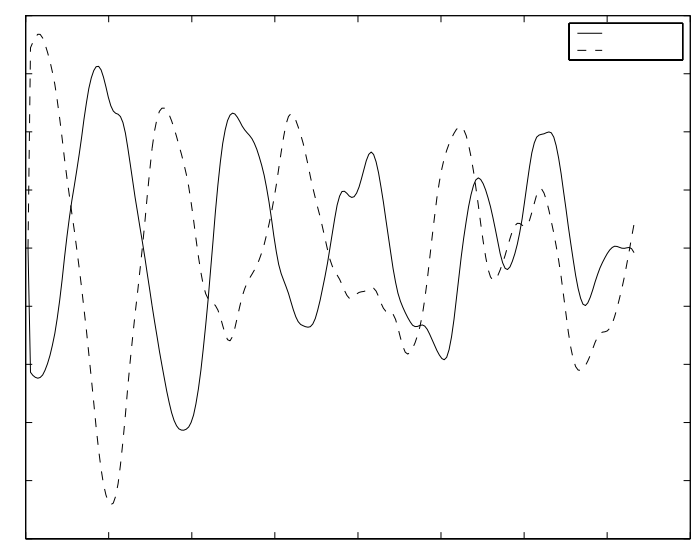

Fig. 10. Comparison of the time records for the target and prediction

Fig. 10 shows a comparison of the target wave record and the prediction made for the first 80 seconds. There would appear to be some identical features to the two records, in particular the average time period of the waves, but overall it is a poor match.

\section{Further work}

The problem with the prediction method as it stands may lie in the assumption that $\phi$ can take a random value. Zhang [2] takes a different approach using a maximum likelihood method to calculate the prediction parameters. Use of this method may lead to a better prediction.

The methods given in Tucker [9] also go further in eliminating spectral defects in the Fourier method which have as yet not been implemented.

Belmont [1] uses an adaptive windowing method to ensure that the wave record being processed by the FFT has no abrupt changes between the beginning and end of the 
record. This methodology would reduce some of the error in generating the phase information required for an accurate prediction.

When the omni-directional prediction has been made more accurate the problem of directional prediction will be tackled. The simulation of a directional wave buoy is already implemented with the analysis of the returned data yet to be completed but, it is expected that an omnidirectional spectrum, $S(f)$, and a set of directional spectrums, $G(\theta, f)$, will be generated. From this data a sequence of wave trains can be produced and propagated to give a prediction at a point near to the origin.

A further avenue of exploration is in the use of data from the wave devices themselves. The devices will be able to record their orientation, relative motion between segments and various electro-mechanical data. From this information it is hoped that wave elevation and surface displacement in the vicinity of the device would be able to be calculated.

The prediction generated from a wave buoy situated near the wave devices could then be checked against the information from the devices, adjusted and then passed to other devices in the farm.

\section{Conclusion}

Initial research into the short term deterministic prediction of wave behaviour has been given and future paths have been laid out. While initial predictions are not great, with some more experimentation the methodology would appear to be useful and to be extendable to directional predictions.

A further course that may be followed if this methodology should fail would be to look closely at one of the third generation wave models [10] to see if they can be reduced to a scale small enough for a single wave farm. The output of a model of the North Western Atlantic at a large scale could then be used to drive this smaller model reinforced with data collected from a directional wave buoy moored in close proximity to the wave device farm. While not giving an accurate prediction of surface elevation, the spectral outputs of these models could be used to feed the devices general estimates of wavelength and wave height, which would then be used to self tune the devices to a prevailing sea state.

The usefulness of these predictions to wave energy devices would enable them to increase efficiency and thereby lower the cost of the electricity produced, hopefully to a level where it will be competitive with current thermal and renewable sources.

\section{References}

[1] M.R. Belmont, E.L. Morris and H.K. Zienkiewicz, "Short term forecasting of the sea surface shape", Int. Shipbuild. Progr., 45, no. 444 (1998) pp. 383-400.

[2] J. Zhang and I. Preslin, "Deterministic decomposition of deep water short-crested irregular waves", Journal of Geophysical Research, (1997) Vol. 102, No. C6, pp 12677-12688.

[3] S.H. Salter, "Wave power", (1974) Nature, Vol. 249, June $21^{\text {st }} 1974$, pp. $720-724$.

[4] J.R. Halliday and D.G. Dorrell, "Review of wave energy and wave generator development in the UK and the rest of the world", IASTED International Conference on Power and Energy Systems, Rhodes, June 2004.

[5] P.G. Davis, M.S. Cloke, K.A. Major, D.I. Page and R.J. Taylor (ed), "Wave Energy", ETSU R-26, ETSU for the Department of Energy, Harwell, 1985.

[6] European Wave Energy Network, "Results from the work of the European Thematic Network on Wave Energy", European Community ERK5-CT-1999-20001, March 2003

[7] Sir G.B. Airy, "Tides and waves", Encyclopaedia Metropolitania, 1845

[8] D.E. Cartwright, in Ocean Wave Spectra, "The use of directional spectra in studying the output of a wave recorder on a moving ship", pp 203-218, Prentice-Hall, Englewood Cliffs, N.J., 1963

[9] M.J. Tucker and E.G. Pitt, "Waves in Ocean Engineering", volume 5 of Elsevier Ocean Engineering Book Series, Elsevier Science Ltd. 2001

[10] N. Booj, R.C. Ris and L.H. Holthuijsen, 1999, "A thirdgeneration wave model for coastal regions, Part I, model description and validation", J. Geoph. Research, C4, 104, pp 7667-7681.

[11] W.J. Pierson and L. Moskowitz, 1964, "A proposed spectral form for fully developed wind seas based on the similarity theory of S.A. Kitaigorodskii”, J. Geophysical Research, 69, pp 5181-5203. 